\title{
ON THE EVOLUTION OF PSEUDOGAMY
}

\author{
Nils Chr. Stenseth and Lawrence R. Kirkendall \\ Department of Biology, Division of Zoology, University of Oslo, \\ P.O. Box 1050, Blindern, 0316 Oslo 3, Norway
}

\author{
AND \\ NANCY MORAN ${ }^{1}$ \\ Museum of Zoology, Division of Biological Sciences, University of Michigan, \\ Ann Arbor, Michigan 48109
}

\begin{abstract}
Summary - A density- and frequency-dependent model for the evolution and maintenance of pseudogamous females is developed and analyzed. Ecological as well as evolutionary aspects of pseudogamy are discussed. Criteria are described for the stable coexistence of sexual females and pseudogamous females under natural conditions. The conditions for invasion of a normal bisexual population by pseudogamous females are less stringent than the conditions for stable coexistence. Hence, we expect that some populations will be characterized by unstable sex ratios over time (with the resulting local extinction due to lack of males) while other populations will be characterized by stable sex ratios over time. If high population sex ratios (i.e., many females to few males) are to be stable, the net population growth rate must be large, and there can be no successful male preference for sexual females.
\end{abstract}

Received August 25, 1983. Revised March 28, 1984

Pseudogamy, or gynogenesis, ${ }^{2}$ is the peculiar form of parthenogenetic reproduction in which females do not produce offspring unless they have mated-despite the fact that paternal genes are not incorporated into the female's progeny (Oliver, 1971). The offspring produced are all females; pseudogamy is therefore a special form of asexual reproduction, and the topic as a whole relates to the problem of understanding sexual reproduction in the neo-Darwinian framework (e.g., Williams, 1975; Maynard Smith, 1978; Bell, 1982).

Pseudogamy occurs in a variety of monocotyledonous and dicotyledonous

\footnotetext{
${ }^{1}$ Current address: Department of Biological Sciences, Northern Arizona University, Flagstaff, AZ 86011 .

${ }^{2}$ Both terms are in current usage. We prefer "pseudogamy" (meaning "false marriage") over "gynogenesis" ("female descent") simply because it better describes the phenomenon. Any form of asexual reproduction necessarily involves an allfemale lineage; the asexual phenomenon we discuss here is distinguished by the necessity for females to copulate with males. "Pseudogamy" also seems to have precedence, having been used at least as early as 1881 by the botanist W. D. Focke.
}

plants (Focke, 1881; Gustafsson, 19461947; Nygren, 1954) and in at least 17 different families of six animal phyla (Hale, 1964; Birky and Gilbert, 1971; Kiester et al., 1981; Bell, 1982). These comprise organisms as diverse as blackberries, citrus trees, meadowgrass, rotifers, nematodes, mites, moths, fish, and salamanders. Many pseudogamous lineages are known to be polyploid (usually triploid) and are known to be of hybrid origin in planthoppers (Drosopoulos, 1978; Booij, 1981), bark beetles (e.g., the Ips tridens complex; Lanier and Oliver, 1966), salamanders (Uzzell and Goldblatt, 1967), and poeciliid fish (Schultz, 1969, 1973). Polyploidy and hybridism seem to characterize pseudogamous plants as well (Gustafsson, 1946-1947; Nygren, 1954).

The presence of such asexual females in their "host" sexual population results in female-biased sex ratios, which can vary dramatically within a species' range of distribution. These biased sex ratios seem to remain stable from year to year in some populations of pseudogamous salamanders (Wilbur, 1971 Table 6), planthoppers (Drosopoulos, 1977; Booij 
and Guldemond, 1984), and moths (Mitter et al., 1979).

Such observations raise interesting ecological and evolutionary questions about the population dynamics of this reproductive habit (Clanton, 1934; Moore, 1976; Mitter and Futuyma, 1977; Kiester et al., 1981). The central questions are, first, under what conditions can pseudogamous females invade a normal sexual population? Second, when pseudogamy is successful, why does not the increasingly female-biased sex ratio ultimately drive the population to extinction? That is, under what conditions does a stable coexistence of pseudogamous and bisexual lineages become possible? And third, what might explain the large sex ratio variation commonly found among populations in which the two female types coexist?

These questions can be addressed empirically through (a) the comparative analysis of mixed populations under a variety of ecological conditions, and (b) experiments in which mixed populations are subjected to manipulations of biotic and abiotic factors. Theoretical analysis of the population dynamics of mixed systems - the subject of this paper-can provide a "roadmap" for such research, by identifying those biological factors likely to be most important, and by providing testable hypotheses.

Two such theoretical analyses have earlier been published (Moore, 1976; Kiester et al., 1981). Moore's (1976) frequency-dependent model is biologically the more comprehensive of the two theoretical treatments of competition between pseudogamous and sexual females. The model of Kiester et al. (1981) is more limited in scope but deals with hermaphroditic as well as dioecious populations. Here, we discuss only the consequences of pseudogamy arising in dioecious organisms; specifically, we extend Moore's analysis by incorporating the effect of density dependence as well as frequency dependence in demographic rates.

\section{THE MODEL}

\section{A Regular Bisexual Population}

Sexual females are assumed to produce broods with a 1:1 sex ratio; it is further assumed that there is no difference in the sexes' survival. Violation of these assumptions will not affect the generality of the results, though the theoretical analysis becomes more difficult. Given the above assumptions, the density of sexual females (as well as the density of males) is given by $x$, and $2 \cdot x$ will the be the total density of the bisexual population. Let $\beta_{1}$ be the expected instantaneous rate of production of adult progeny by mated females, and $\mu_{1}$ the corresponding adult mortality rate. Finally, the potential number of mated females per male is given by $\alpha$, which must be less than or equal to the ratio of females to males. Thus, in a pure sexual population with $1: 1$ breeding adult sex ratio, $\alpha \leq 1$. The primary determinants of $\alpha$ for a given population will be the efficiency of the mate-location system together with physical or resource limitations on how many females a male can potentially inseminate or host in his territory.

Utilizing these assumptions and quantities, it follows that -in the case of continuous reproduction - the dynamics of the female half of the population are given by

$$
d x / d t=.5 \cdot \beta_{1} \cdot x \cdot \alpha-\mu_{1} \cdot x .
$$

Verbally, equation (1) says that change over time in number of adult females results from the expected production of adult progeny by each mated female $\left(\beta_{1}\right)$ times the number of mated females $(x \cdot \alpha)$, reduced by the total female mortality $\left(\mu_{1} \cdot x\right)$.

\section{A Mixed Bisexual-Pseudogamous Population}

Let $y$ be the density of pseudogamous (or asexual) females, and let $\beta_{2}$ be the rate at which pseudogamous females produce adult offspring - all of which are themselves female. The specific mortality rate 
for the pseudogamous form is $\mu_{2}$. Sexual and pseudogamous females could differ in their success at obtaining sperm, if access to males were somehow limiting. In order to incorporate such an effect we introduce $p(0 \leq p \leq 1)$ and $q(q=1-$ $p$ ) such that $p / q$ is the relative pairing success of sexual females: then, $p / q=1$ ( $p=q=.5$ ) represents random mating, whereas $p>.5$ indicates that sexual females are disproportionately more likely than pseudogamous females to become inseminated. Greater pairing success for sexuals could arise if (a) males mate more readily with sexual than with pseudogamous females, or (b) if some inbreeding occurs. Finally, $\alpha$ is defined as before, but in a population including pseudogamous females $\alpha$ can be greater than 1 , since the number of mated females can exceed the number of males. However, $\alpha$ must always be less than or equal to the overall ratio of females to males in the population: $\alpha \leq(x+y) / x=1+y / x$. Note that if all females obtain sperm $(\alpha=1+$ $y / x), p / q$ must equal 1 , though $p$ and $q$ are otherwise assumed to be independent of $\alpha$.

The dynamics of the two types of female in a mixed population are given by

$$
\begin{aligned}
d x / d t= & .5 \cdot \beta_{1} \cdot x \cdot \alpha \\
& \cdot(p \cdot x /(x \cdot p+y \cdot q)) \\
& -\mu_{1} \cdot x \\
d y / d t= & \beta_{2} \cdot x \cdot \alpha \\
& \cdot(q \cdot y /(x \cdot p+y \cdot q)) \\
& -\mu_{2} \cdot y .
\end{aligned}
$$

The quantities $p \cdot x /(x \cdot p+y \cdot q)$ and $q \cdot y /(x \cdot p+y \cdot q)$ represent the potential proportions of mated sexual and pseudogamous females; multiplying each quantity by $\alpha$ gives the actual proportions mated (as would be observed in the field).

\section{Density and Frequency Dependence}

Our model should properly be described as a mixed density-dependent and frequency-dependent model. The basic quantities of the model are density dependent: for a fixed frequency of sexuals to asexuals $(x / y)$, the specific population growth rate functions, $W_{x}=(1 / x) \cdot d x / d t$ and $W_{y}=(1 / y) \cdot d y / d t$ (i.e., their individual fitnesses), will be functions of density. Frequency dependence arises from the proportional mating success terms, $p \cdot x /(x \cdot p+y \cdot q)$ and $q \cdot y /(x \cdot p+y \cdot q)$; for a fixed density at breeding sites, $z=2 \cdot x$ $+y$, the specific population growth rate functions will be functions of the frequency of sexuals to asexuals. Thus, changes in the frequency of sexual females at a constant density will alter the relative proportion of sexual females mated, when $p / q \neq 1$. Altogether, this means that the fitnesses for sexual females and for pseudogamous females are given as $W_{x}=W_{x}(z, x, y, x / y)$ and $W_{y}=$ $W_{y}(z, x, y, x / y)$.

This way of modelling evolutionary processes-i.e., emphasizing the ecological aspects of the process-is different from what is commonly seen in modern literature on evolution and evolutionary ecology (e.g., Roughgarden, 1979). Notice, however, that our approach is not new: it was, for example, applied by Lawlor and Maynard Smith (1976). Indeed, it was the approach favored by Kostitzin (1937), one of the pioneers in the field of population biology.

At the end of this paper we relax the assumption that $z=2 x+y$ is an appropriate expression for density; for example, the two forms may exploit different niches (e.g., Vrijenhoek, 1979; Christensen, 1980) and therefore compete primarily or solely for sperm. In such a case $\beta_{1}$ and $\mu_{1}$ should depend primarily on $x$, $\beta_{2}$ on $y$, and $\mu_{2}$ on both $x$ (due to competition for matings) and $y$ (due to competition for resources).

\section{The Form of the Functions}

$\beta_{\mathrm{i}}$. - The net specific reproductive rate function, $\beta_{i}=\beta_{i}(z)$, is generally a decreasing function of density-possibly with the exception of lower densities where it initially may increase. Most likely, $\beta_{1}(0)$ and $\beta_{2}(0)$ are different; unfortunately there is no a priori reason to decide which one should be larger. Figure $1 \mathrm{~A}$ depicts a likely form of $\beta_{i}$. 
A
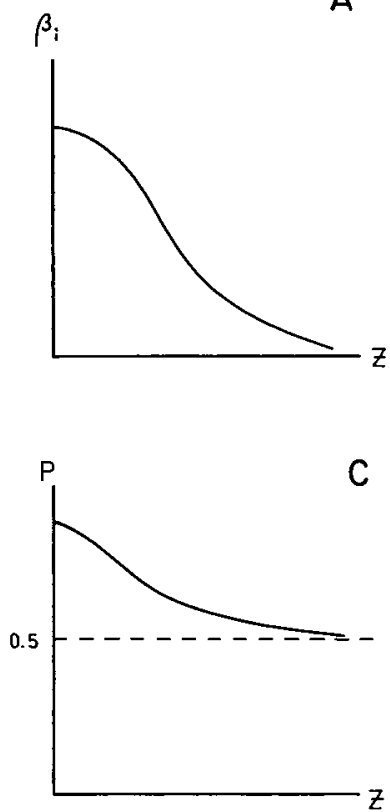

B
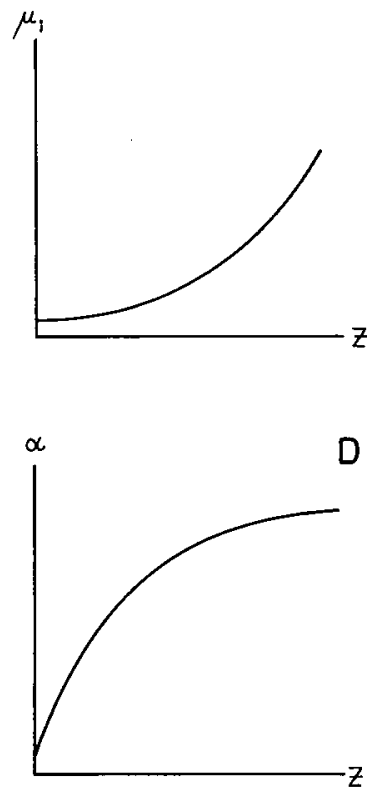

Fig. 1. The forms assumed in our model for the demographic $\left(\beta_{i}\right.$ and $\left.\mu_{i}\right)$ and behavioral $(\alpha, p$ and $q)$ parameters as functions of density $(z) .1 \mathrm{~A}: \beta_{i}$, the specific reproductive rate. $1 \mathrm{~B}: \mu_{i}$, the specific mortality rate. $1 \mathrm{C}: p$, differential success at obtaining sperm, where $p=.5$ indicates random mating with respect to female type and $p>.5$ disproportionately greater success for sexual females (assortative mating favoring sexuals). 1D: $\alpha$, the potential ratio of mated females to males (see text).

$\mu_{\mathrm{i}}$ - - The mortality rate increases with density $(z)$. In addition, $\mu_{i}$ is likely to increase as the frequency of asexuals $(y / x)$ rises to the point where it becomes more and more difficult to find a mate and acquire sperm. Under such conditions it may-for a given density $(z)$ - be relatively easier for sexual than for pseudogamous females to find a male, and $\mu_{2}$ might therefore be larger than $\mu_{1}$. Such frequency dependence for $\mu_{i}$ would, however, not affect the conclusions drawn below. Figure 1B depicts a reasonable form for $\mu_{i}$.

p.-A density-dependent relative advantage for sexuals in obtaining sperm (i.e., $p>.5$ or $p / q>1$ ) could arise from either passive or active mate selection by males. Thus, at low densities, sexual females are more likely to be near a male (e.g., a brother) than are asexual females, implying that inbreeding could provide a high value for $p$. Such an advantage would presumably be small or non-existent at high densities. Such a process is "passive" in that male discrimination between sexual and pseudogamous females is not required. Similarly, where male preference for pairing with sexual females has evolved, males may be best able to actively discriminate between females at low densities - at least if mating systems tend to break down at higher densities (e.g., McKay, 1971; Moore and McKay, 1971).

Again, frequency dependence would, most probably, have an effect similar to density dependence. In cases where inbreeding can lead to $p>.5$, low sexual frequency (i.e., high $y / x$ ) has the same effect as low density: sexual females are more likely than pseudogamous ones to be near the few males, an effect which would tend to disappear as the frequency of sexuals in the population increases. The effect of frequency dependence in an active mate selection system is less clear. On the one hand, territorial systems which exclude some males from breeding can break down at high male density (i.e., 
at low $y / x$ ), leading to greater mating success for nondiscriminating satellite males (as occurs in mixed Poeciliopsis populations; Moore and McKay, 1971). On the other hand, it is conceivable that systems in which females court males to gain admittance to harems (as in, for example, pseudogamous Ips bark beetles; Lanier and Oliver, 1966; Bakke, 1968) might cease to function under extremely high female to male ratios (i.e., high $y / x$ ). In the first case it is a high density of sexuals (i.e., of males) that leads to low $p / q$, while in the second it is a high density of asexuals (i.e., of females) that does so.

In the following, we will assume that the frequency-dependent effects on $p$ (and $q)$ are in the same direction as the density dependence discussed above (see Fig. 1C). However, in reality the relationship between frequency and density and their net effect on $p / q$ may turn out to be considerably more complex than we have assumed it to be. Given actual data on these dependencies, our model could, however, easily be analyzed for such more complex situations.

$\alpha .-$ The average number of matings per male will be affected primarily by the ratio of pseudogamous to sexual females, $y / x$. We expect $\alpha$ to increase initially, as the frequency of asexuals increases, but to level off as it reaches an upper limit defined by either male potency or resource availability. Again, we assume a positive relationship between $y / x$ and density, $z$, and therefore between $\alpha$ and $z$ (Fig. 1D). A similar positive correlation between $\alpha$ and $z$ would also occur-independently of $y / x$ ratio-if mate location was more efficient at higher densities.

\section{$\theta_{i}$, An Auxiliary Function}

The quantities $\alpha$ and $\beta_{i}$ always co-occur as a product in (1) and (2). For mathematical convenience-as well as for simplifying the following discussion-we discuss the properties of $\theta_{i}\left(\theta_{i}=\beta_{i} \cdot \alpha\right)$, which is actually net offspring per male. We thereby incorporate birth rate and potential females per male into this one quantity, which we assume to decrease with increasing density $z$ (Tanner [1966] and Pimm [1983] are relevant) with the possible exception of the lower values of $z$.

\section{INVASIBILITy of a Normal Sexual Population by Pseudogamous FEMALES \\ Ecological Invasion}

Let $\hat{x}$ be the equilibrium density of sexual females in the absence of asexual females. From (1) we have

$$
.5 \cdot \alpha(2 \cdot \hat{x}) \cdot \beta_{1}(2 \cdot \hat{x})=\mu_{1}(2 \cdot \hat{x}),
$$

which can be written as

$$
\alpha(2 \cdot \hat{x}) \cdot \beta_{1}(2 \cdot \hat{x}) / \mu_{1}(2 \cdot \hat{x})=2 .
$$

By substituting $x=\hat{x} \cdot(1+\Delta x)$ into (1) and requiring that $d(\Delta x) / d t<0$, it may easily be seen that the equilibrium given by $\hat{x}$ will be asymptotically stable if $d\left(\alpha \cdot \beta_{1}\right) / d z<2 \cdot d \mu_{1} / d z$. From the stated assumptions for $\beta_{1}, \alpha$ and $\mu_{1}$, this condition is quite likely to be satisfied (see Figs. 1A and B). If a pseudogamous female appears through mutation in-or immigration into-a sexual population which is at this equilibrium, it can invade the sexual population and become established if

$$
\begin{aligned}
\alpha(2 \cdot \hat{x}) \cdot\left[\beta_{2}(2 \cdot \hat{x}) / \mu_{2}(2 \cdot \hat{x})\right] & \\
\cdot & {[q(2 \cdot \hat{x}) / p(2 \cdot \hat{x})]>1, }
\end{aligned}
$$

where the argument in these functions, $2 \cdot \hat{x}$, is a good approximation to $2 \cdot \hat{x}+y$ since $y \approx 0$; as usual, mutant or immigrating asexual females are assumed to be rare initially.

We may now rewrite (5) by utilizing (4) to obtain

$$
\begin{gathered}
2 \cdot\left[\beta_{2}(2 \cdot \hat{x}) / \mu_{2}(2 \cdot \hat{x})\right] \cdot[q(2 \cdot \hat{x}) / p(2 \cdot \hat{x})] \\
>\beta_{1}(2 \cdot \hat{x}) / \mu_{1}(2 \cdot \hat{x}),
\end{gathered}
$$

which, since we have assumed $p \geq .5$ (see Fig. 1C) and hence $q / p \leq 1$, gives us the following condition for invasibility:

$$
2 \cdot \beta_{2}(2 \cdot \hat{x}) / \mu_{2}(2 \cdot \hat{x})>\beta_{1}(2 \cdot \hat{x}) / \mu_{1}(2 \cdot \hat{x}) .
$$

A bisexual population can then be invaded by a pseudogamous mutant or immigrant female if the latter is at least half as "fit" as the sexual "host." This is, of 
TABLE 1. Combinations of conditions under which local stability of the equilibrium $\left(x^{*}, y^{*}\right)$ can occur for (2), using the Routh-Hurowitz Criterion (Appendix $B$ ). The four cases considered here are not mutually exclusive, nor do they represent all possible combinations of conditions. $\left(\lambda_{1}\right.$ and $\lambda_{2}$ are the two eigenvalues of the system-see Appendix B.)

\begin{tabular}{|c|c|}
\hline Case & $\begin{array}{l}\text { Mathematical conditions on the functions } \\
\text { (evaluated at equilibrium) }\end{array}$ \\
\hline I & $\begin{array}{l}\theta_{1} \approx \theta_{2} \text { or } \theta_{1}>\theta_{2} \\
\quad d \theta_{1} / d z \approx d \theta_{2} / d z \text { or } d \theta_{1} / d z<d \theta_{2} / d z \\
\mu_{1} \approx \mu_{2} ; d \mu_{1} / d z \approx d \mu_{2} / d z \\
2 \cdot q>p(\text { i.e., } p<2 / 3) ; d p / d z \leq 0\end{array}$ \\
\hline II & $\begin{array}{l}\theta_{1}>\theta_{2} \text { and } d p / d z<0 \\
\text { [The larger }\left(p \cdot x^{*}+q \cdot y^{*}\right) \text { is (i.e., the } \\
\text { higher the overall density), the clos- } \\
\text { er to zero can } d p / d z \text { be and still be } \\
\text { compatible with local stability of } \\
\text { (2).] }\end{array}$ \\
\hline III & $\begin{array}{l}2 \cdot \theta_{2}>\theta_{1}>\theta_{2} ; d \theta_{1} / d z \approx d \theta_{2} / d z \\
d \mu_{1} / d z \approx d \mu_{2} / d z \\
p \approx .5 \text { and } d p / d z \approx 0\end{array}$ \\
\hline IV & $\begin{array}{l}d \theta_{1} / d z \ll 0 ; d \theta_{2} / d z \ll 0 \\
\quad\left(\text { making } \lambda_{1}+\lambda_{2}<0\right) \\
\theta_{1} \leq \theta_{2}\left(\text { guaranteeing } \lambda_{1} \cdot \lambda_{2}>0\right)\end{array}$ \\
\hline
\end{tabular}

course, the commonly derived condition for the invasion of a sexual population by an asexual mutant form (e.g., Maynard Smith, 1978; Bell, 1982). We have derived this result because we need (7) for the further analysis of our model.

\section{Condition For Sexual Reproduction Being ESS}

The opposite inequality to that given by (6) provides the condition for sexual reproduction being an Evolutionarily Stable Strategy (or ESS; see Maynard Smith, 1982; Reed and Stenseth, 1984). That is, if the opposite inequality of (6) is satisfied for any possible mutant asexual strategy (i.e., for asexuals with any possible combinations of $\beta_{2}$ and $\mu_{2}$ ), then invasion is impossible and sexuality will be an ESS.

\section{Stable Coexistence of the Two FEMALE TyPES}

\section{General Properties of the Equilibrium}

Let $x^{*}$ and $y^{*}$ denote the equilibrium of the two types of females. By definition, a stable coexistence equilibrium with both types present requires that both $x^{*}$ and $y^{*}$ are finite and positive numbers different from zero; this is equivalent to requiring that $z^{*}=2 \cdot x^{*}+y^{*}$ be a finite positive quantity.

Setting $d x / d t=d y / d t=0$, we find after minor algebraic manipulation that

$$
\begin{gathered}
.5 \cdot\left[\beta_{1}\left(z^{*}\right) / \beta_{2}\left(z^{*}\right)\right] \cdot\left[p\left(z^{*}\right) / q\left(z^{*}\right)\right] \\
=\mu_{1}\left(z^{*}\right) / \mu_{2}\left(z^{*}\right)
\end{gathered}
$$

must hold for $z^{*}$ to be a finite number.

Special Cases. - First, let $\left[\beta_{2}(z) / \beta_{1}(z)\right]$. $\left[\mu_{1}(z) / \mu_{2}(z)\right] \equiv 1$ for all values of $z$. This will be true, for example, if (i) $\beta_{1} \equiv \beta_{2}$ and $\mu_{1} \equiv \mu_{2}$, or (ii) $\beta_{1} \equiv \mu_{1}$ and $\beta_{2} \equiv \mu_{2}$ (and thus $\beta_{i}$ and $\mu_{i}$ density-independent) at all densities. Since, at equilibrium, $p / q$ must now equal 2 to satisfy (8), and we assume $d(p / q) / d z<0$ (Fig. 1C), stable coexistence then requires strong active or passive mate discrimination favoring sexual females to counterbalance the twofold reproductive advantage to pseudogamous females. Thus, if $p(0) / q(0)>2$, a stable coexistence equilibrium will be reached at that density at which $p / q=2$. Without density dependence in $p$, it is extremely unlikely that (8) could be satisfied: $p / q$ must exactly equal two.

Now, let us assume that, for the two types of females, the fecundity functions are identical (i.e., $\beta_{1} \equiv \beta_{2}$ ) and that $p / q=$ 1. Then, we may write (8) as

$$
.5 \cdot \tau\left(z^{*}\right)=1 \text {, }
$$

where $\tau(z)=\mu_{2}(z) / \mu_{1}(z)$. (A similar argument, using $\phi(z)=\beta_{1}(z) / \beta_{2}(z)$, can of course be given by assuming $\mu_{1} \equiv \mu_{2}$; substitute in this case $\tau$ by $\phi$ in (9).) If $\tau(0)<2$ and if $d \mu_{2} / d z>2 \cdot d \mu_{1} / d z$, both evaluated at $z^{*}$, stable coexistence at $z^{*}$ is possible. Altogether, this implies that stable coexistence requires that the rate of mortality at low density be higher for the sexual form than for the asexual form and that the asexual form be most sensitive to increasing density. On the other hand, $\tau(0)>2$ requires that the sexual form be more sensitive to increasing density.

The General Case.-In general, the stability properties of model (2) may be found by analyzing its Jacobian matrix (defined in Appendix A); this analysis is 
summarized in Appendix B and Table 1. From this we see that, in general, strong density dependence in $\theta_{i}$ (i.e., $d \theta_{i} / d z \ll$ 0 -where $\left.\theta_{i}=\beta_{i} \cdot \alpha\right)$ and the mortality rates (i.e., $d \mu_{i} / d z \gg 0$ ) will contribute to stabilizing ecological equilibrium $\left(x^{*}, y^{*}\right)$. Furthermore, stability can easily result if the success of sexual females in acquiring matings is density-dependent (i.e., $d p /$ $d z<0$ ), given that the specific reproductive rate of sexual females is higher than that of pseudogamous females at equilibrium and thus $\theta_{1}>\theta_{2}$ (see Case II in Table 1). Finally, stability can easily result if the probability of success of sexual females in acquiring matings at equilibrium is below $2 / 3$. (Recall that $p(0) / q(0)>$ 2 and that $d(p / q) / d z<0$. This is then consistent with the assumption that $1 / 2 \leq$ $p\left(z^{*}\right)<2 / 3$, as $p\left(z^{*}\right) / q\left(z^{*}\right)$ will then be less than 2.)

When $\theta_{1}>\theta_{2}$ at equilibrium $\left(x^{*}, y^{*}\right)$, density dependence of $p$ can provide stability but will not necessarily do so (Case III in Table 1); nor is density dependence in demographic rates sufficient to guarantee stability (Case II in Table 1). Notice also that stability can easily result even when the success of sexual females in acquiring matings is density-independent (Case IV in Table 1). Case I in Table 1 is more complicated but nevertheless quite likely to be realized under natural conditions.

In summary, we may then say that, given that everything else is the same, the following features will contribute to stabilizing the ecological equilibrium:

(i) $\quad d \theta_{i} / d z \ll 0$;

(ii) $\quad d \mu_{i} / d z \gg 0$;

(iii) $d p / d z<0$, given that $\theta_{1}>\theta_{2}$; or

(iv) $p<2 / 3$.

\section{The Population Sex Ratio}

To examine the equilibrium sex ratio of the population,

$$
S R^{*}=1+y^{*} / x^{*},
$$

we must first compute the change over time in the ratio of sexual to asexual females, $v=x / y$. Thus, $(1 / v) d v / d t=(1 / x)$. $d x / d t-(1 / y) \cdot d y / d t$, substituting from (2) and using $\theta_{i}=\beta_{i} \cdot \alpha$,

$$
\begin{aligned}
d v / d t= & {\left[.5 \cdot \theta_{1} \cdot(p \cdot v /(v \cdot p+q))\right.} \\
& -\theta_{2} \cdot(q \cdot v /(v \cdot p+q)) \\
& \left.-\left(\mu_{1}-\mu_{2}\right)\right] \cdot v .
\end{aligned}
$$

The equilibrium ratio, $v^{*}=x^{*} / y^{*}$, is then found by solving $d v / d t=0$, with all functions evaluated at $\left(x^{*}, y^{*}\right)$ :

$$
\begin{aligned}
v^{*}=x^{*} / y^{*}= & \left(\mu_{1}-\mu_{2}\right) \\
& \cdot q /\left[.5 \cdot \theta_{1} \cdot p-\theta_{2} \cdot q\right. \\
& \left.-\left(\mu_{1}-\mu_{2}\right) \cdot p\right] .
\end{aligned}
$$

Analyzing the stability of (11), we find that $v^{*}=x^{*} / y^{*}$ is asymptotically stable if $d \theta_{1} / d z<0, d \mu_{i} / d z>0$, and $d p / d z<0$; these requirements correspond directly to those found to guarantee the stability of $\left(x^{*}, y^{*}\right)$ and summarized in Table 1 . Furthermore, $v^{*}>.5$ will be stable if $z^{*}$ is large and $p / q>1$. On the other hand, $v^{*}<.5$ will be stable if $z^{*}$ is small and $p / q<1$. Hence, we see that mate selectivity in males or inbreeding (i.e., $p / q>$ 1 as explained above) will stabilize the $x^{*} / y^{*}$ ratio at a high value in a large population; this will correspond to a dense population with relatively few pseudogamous females.

The ratio of the two types of females may further be studied by evaluating the isoclines of (2). These are implicitly defined by

$$
\begin{aligned}
y / x= & \left(.5 \cdot\left(\beta_{1} \cdot \alpha\right) / \mu_{1}-1\right) \\
& \cdot p /(1-p)
\end{aligned}
$$

for the $x$-isocline (Fig. 2A), and

$$
y / x=\left(\beta_{2} \cdot \alpha\right) / \mu_{2}-p /(1-p)
$$

for the $y$-isocline (Fig. 2B). Notice that, as functions of $x$ and $y$, these isoclines will generally not intersect if the basic quantities $\left(\beta_{i}\right.$ and $\mu_{i}, \alpha$ and $\left.p\right)$ entering (2) are density-independent; however, even then we can find values of $p$ so that the isoclines intersect.

The equilibrium $\left(x^{*}, y^{*}\right)$ of model (2) is found by the intersection of (13) and (14) (see Fig. 2C). Equation (13) (or (14)) combined with (8) gives

$$
y^{*} / x^{*}=\left(\beta_{2} / \mu_{2}\right) \cdot\left(\alpha-2\left(\mu_{1} / \beta_{1}\right)\right)
$$


A

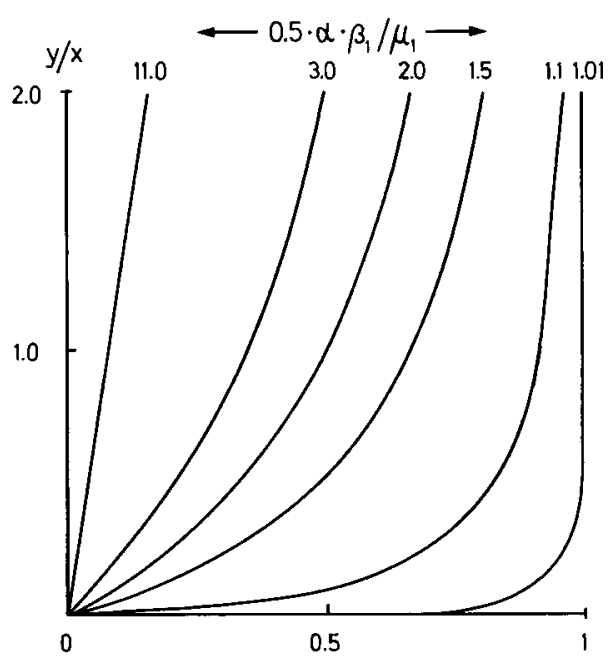

B

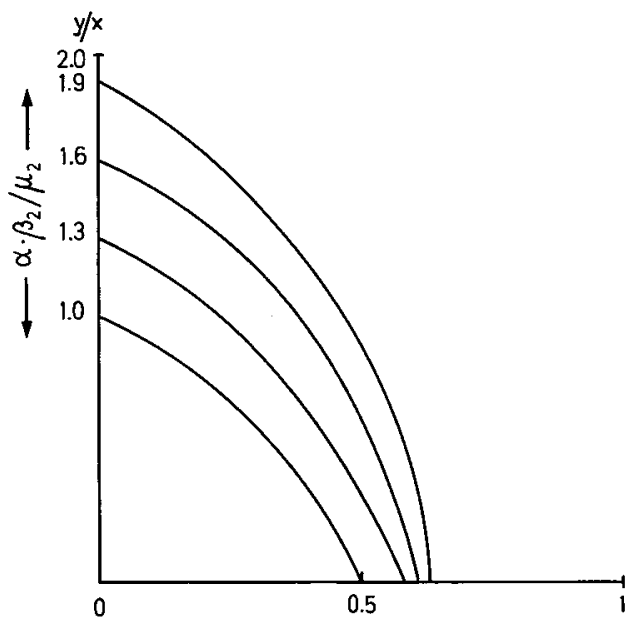

where all functions are evaluated at equilibrium.

Knowing the equilibrium $y^{*} / x^{*}$ ratio, we can find the equilibrium population sex ratio, $S R^{*}$ by (10). From Figure $2 \mathrm{C}$ we then see that a high $S R^{*}$ requires high $\left(\beta_{1} \cdot \alpha\right) / \mu_{1}$ and high $\left(\beta_{2} \cdot \alpha\right) / \mu_{2}$; from (15), we then see that if $\alpha>2 \cdot\left(\mu_{1} / \beta_{1}\right), \beta_{2} / \mu_{2}$ must be high in order to obtain a high $S R^{*}$. Such might be the case for species with high population growth potential. Furthermore, $p$ must be low in order to produce a high $S R^{*}$ : in particular, we can see from Figure $2 \mathrm{C}$ that if $p \approx .5,\left(\beta_{i} \cdot \alpha\right) /$
C

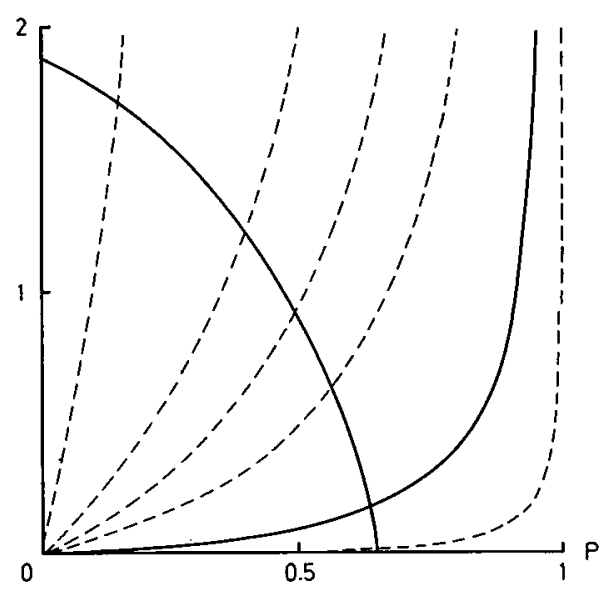

FIG. 2. Implicit definitions of the isoclines as functions of the demographic $\left(\beta_{i}, \mu_{i}\right)$ and behavioral $(\alpha, p)$ terms of the model. In $2 \mathrm{~A}$, the $x$-isocline is defined implicitly by holding $y$ constant and calculating the appropriate $y / x$ ratio for each value of $p$, such that $d x / d t=0 ; 2 \mathrm{~B}$ depicts the similarlyconstructed $y$-isocline $(d y / d t=0)$. In $2 \mathrm{C}$, the intersection of the solid curves indicates the stable $y^{*} /$ $x^{*}$-ratio, for the $x$-and $y$-isoclines arising from $\beta_{1} \approx$ $\mu_{1}$ and $\beta_{2} \approx \mu_{2}$. (Dashed curves represent the other $x$-isoclines from $2 A$.) In such a population, stability requires passive or active mate discrimination in favor of sexual females.

$\mu_{i}(i=1,2)$ needs to be very high. This is indeed consistent with the condition (see equation (5)) for invasibility of pseudogamous females into a regular sexual population.

Moore (1976) related the proportion of asexual females $(y / x)$ to what he called relative primary fitness of asexual females; this relative primary fitness ratio, $R$, seems - in our terminology-most appropriately to be given as

$$
R=\left(\beta_{2}-\mu_{2}\right) /\left(\beta_{1}-\mu_{1}\right) .
$$

If we rewrite (15) by using $R$, we obtain 


$$
\begin{aligned}
y^{*} / x^{*}= & {\left[\left(R / \mu_{2}\right) \cdot\left(\beta_{1}-\mu_{1}\right)+1\right] } \\
& \cdot\left(\alpha-2 \cdot\left(\mu_{1} / \beta_{1}\right)\right) .
\end{aligned}
$$

As can be seen, we obtain the same general association between $y^{*} / x^{*}$ and $R$ as Moore (1976) did. Furthermore, we see that strongly female-dominated populations will be favored by one or both of the following conditions: i) a high degree of polygamy (i.e., high $\alpha$ occurring at high total population density; see Fig. 1D), and ii) high fecundity $\left(\beta_{i}\right)$ relative to adult mortality $\left(\mu_{i}\right)$ of both female types. This will occur under conditions of strong competition for breeding resources. Conversely, the equilibrium proportion of asexual females will be relatively low if mean production of adult progeny by mated females is approximately equal to the adult mortality rate and if the potential number of mated females per male is low.

\section{Interdependence of the Basic Functions at Equilibrium}

Note that $\beta_{1}$ and $\mu_{1}$ cannot be changed independently of each other (nor can $\beta_{2}$ and $\mu_{2}$ ); in particular the following relations must be satisfied at equilibrium:

$$
\begin{aligned}
\mu_{1}\left(z^{*}\right) / \beta_{1}\left(z^{*}\right)= & .5 \cdot \alpha\left(z^{*}\right) \\
& \cdot p\left(z^{*}\right) \cdot x^{*} / \\
& {\left[p\left(z^{*}\right) \cdot x^{*}\right.} \\
& \left.+q\left(z^{*}\right) \cdot y^{*}\right]
\end{aligned}
$$

and

$$
\begin{aligned}
\mu_{2}\left(z^{*}\right) / \beta_{2}\left(z^{*}\right)= & .5 \cdot \alpha\left(z^{*}\right) \\
& \cdot q\left(z^{*}\right) \cdot x^{* /} \\
& {\left[p\left(z^{*}\right) \cdot x^{*}\right.} \\
& \left.+q\left(z^{*}\right) \cdot y^{*}\right] .
\end{aligned}
$$

Additionally, the equilibrium sex ratio of a given population is related to the number of mates per male $(\alpha)$.

Caution must be exercised when considering why populations (or species) might differ in the proportion of asexual females at equilibrium. Equations (12)(15) suggest, for instance, that asexual females will predominate when the breeding success per sexual female $\left(\beta_{1}\right)$ is high relative to adult mortality of sexual fe- males $\left(\mu_{1}\right)$, and when there is no assortative mating or when assortative mating favors pseudogamous females (i.e., $p \leq$ $.5)$. This is so simply because of the relationship between the variables in (2) when equilibrium is assumed; the $\beta_{1}$ function can, at equilibrium, only be high when $\mu_{1}$ also is relatively high. In particular, if the population is at (and remains at) equilibrium, $\beta_{1}$ will be high relative to $\mu_{1}$ when mating success of sexual females is adversely affected by the presence of many pseudogamous females (i.e., when $y$ is high) and/or if $\alpha$ is low.

\section{Sexual and Asexual Females Exploiting Different Niches}

Where pseudogamous lineages are hybrid or polyploid, it seems unlikely that sexual and pseudogamous females utilize identical niches, as we have assumed up to this point. Therefore, we now relax the assumption that the demographic functions $\left(\beta_{i}\right.$ and $\left.\mu_{i}\right)$ are functions of both $x$ and $y$. When two types of females utilize more-or-less different niches, $\beta_{1}$ and $\mu_{1}$ will mainly depend on $x$, and $\beta_{2}$ mainly on $y$, and $\mu_{2}$ on some combination of $x$ and $y$ (but presumably on some density less than $2 \cdot x+y$ ).

In general we have that $\beta_{1}(x+a \cdot y)$ and $\mu_{1}(x+a \cdot y)$ where $0 \leq a \leq 1$ and $\beta_{2}\left(b_{1}\right.$. $x+y)$ and $\mu_{2}\left(b_{2} \cdot x+y\right)$ where $0 \leq b_{1} \leq$ 1. Presumably $a=b_{1}$; hence, we assume so in the following discussion; it is difficult to say anything about the magnitude of $b_{2}$ without data. If the two female types utilize the same niches, then $a=$ $b_{1}=1$; if they utilize completely different niches, then $a=b_{1}=0$. Assuming that males compete with sexual females for resources, (6) - the condition for invasibility - can, in the case of complete nicheseparation, be rewritten as

$$
\begin{gathered}
2 \cdot\left[\beta_{2}(0) / \mu_{2}\left(b_{2} \cdot \hat{x}\right)\right] \cdot(q / p) \\
>\beta_{1}(2 \cdot \hat{x}) / \mu_{1}(2 \cdot \hat{x}) .
\end{gathered}
$$

Hence, pseudogamous females can more easily invade a regular sexual population in the case of niche differentiation. Analysis demonstrates, however, that stable coexistence becomes more difficult in this 
latter case; the sexual population may now more easily be driven to extinction. Stronger competition for males ensues when relaxation of resource competition with sexuals leads to a more rapid increase of asexual females; this is especially so when demographic rates are strongly density-dependent.

Thus, in the case of complete niche overlap, both forms will experience the same female density ( $x$ plus $y$ ) when competing for resources or for sperm. At the other extreme, with no resource competition between types, reproductive rates of each type will be determined by each type's own density ( $x$ or $y$ ): sexual females will experience lower birth rates and higher mortality rates arising from the relatively high sexual female density, while an invading pseudogamous lineage will have the relatively high birth rates and low mortality rates accompanying low density. These effects - to the benefit of the asexuals - come in addition to the two-fold advantage of producing only female offspring. Sexual females will then rapidly face stronger and stronger competition for sperm which could be further amplified by density dependence of $p$. Under niche-separation, then, sexual females will consistently experience stronger competition for males at any given male density than they would under complete niche overlap.

\section{Discussion AND CONCLUSION}

We began by asking why invading pseudogamous lineages do not drive the sperm-donating lineage (and thus themselves) extinct. In developing a model to answer this question, we first assumed males to be limiting, an assumption known to be true for Poeciliopsis (e.g., McKay, 1971), Ambystoma salamanders (Wilbur, 1971), Muellerianella planthoppers (Booij and Guldemond, 1984), and Ips acuminatus bark beetles (Kirkendall, unpubl. observ.), and not known to be false for any species group with pseudogamous females. We then analyzed the properties of the population's equilibrium density; indeed notice that the model only applies to the invasibility of sexual populations at equilibrium and to the ecological stability of mixed populations under that assumption.

The analysis of the model suggests a variety of relationships (Table 1) between birth rates, mortality rates, and passive or active male discrimination against pseudogamous females which could lead to stable coexistence of the competing female types. In general these relationships most readily result in stable coexistence when these variables are strongly density (and frequency) dependent. Finding a lack of density dependence in a stable mixed population would be a clear indication that our model is inadequate.

Why are population sex ratios so variable: what general factors lead to such dramatic differences in the success of sexual females relative to pseudogamous females? Sex ratios for populations of $\mathrm{Am}$ bystoma laterale and $A$. "tremblayi" (a pseudogamous triploid hybrid) vary from 2:1 (females : male) to 52:1 (Wilbur, 1971 Table 6); in mixed populations of Ips acuminatus, from $1.4: 1$ to more than 30:1 (Bakke, 1968); and in Muellerianella fairmairei and its triploid pseudogamous hybrid, from 1.1:1 to 50:1 (Booij and Guldemond, 1984).

Our model suggests answers to this question. It predicts that researchers will find the strongest female bias in populations with highest $\alpha$ (harem size, or male potency) or with high fecundity and juvenile survivorship $\left(\beta_{i}\right)$ relative to mortality $\left(\mu_{i}\right)$.

The causes underlying variation in $\alpha$ may well differ from species to species. One possibility is interpopulation variation in mate locating success, or in mate location systems, both of which in turn may be determined by population density (Fig. 1) or distribution of resources. In stable populations in which $\beta_{i}\left(z^{*}\right)>$ $\mu_{i}\left(z^{*}\right)$ the "extra" mortality must arise from females not mating and giving birth, implying stronger competition for sperm than in populations with $\beta_{i}\left(z^{*}\right) \approx \mu_{i}\left(z^{*}\right)$.

Altogether we expect pseudogamy to occur more frequently in $r$-selected 
species (or populations) than in $K$-selected species (or populations) (sensu Pianka, 1970).

Finally, we have demonstrated that, when pseudogamous and sexual females occupy different niches, it is easier for pseudogamous females to invade a sexual population but it is also more likely that they will drive the population (and themselves) to extinction. Nevertheless, what little is known about pseudogamous forms suggests that many do, indeed, utilize resources differently from their bisexual counterparts. Triploid hybrid Poeciliopsis 2 monacha-lucida lineages differ in habitat preference, feeding behavior, and mouthpart morphology from both parent species, $P$. monacha and $P$. lucida; furthermore, pseudogamous lineages differ from each other in these (as well as other) traits (Schultz, 1977; Vrijenhoek 1978, 1979). Vegetation structure and climate are correlated with the relative success of pseudogamous vs. "host" sexual Muellerianella fairmairei planthoppers in different populations, though they shared the same host plant species; in Denmark and at higher elevations in central Europe, pseudogamous females even utilize completely different host plant species from the sperm donor, which in this case is $M$. brevipennis (Booij and Guldemond, 1984). Pseudogamous Muellerianella forms are triploid and thought to be hybrids between $M$. fairmaire $i$ and $M$. brevipennis (Drosopoulos, 1977, 1978; Booij, 1981). Most other examples of pseudogamy also seem to be interspecific hybrids, polyploid, or both. Polyploidy alone is generally considered to result in ecologically significant morphological and physiological divergence from the diploid ancestor (White, 1978; see also various papers in Lewis, 1980). Thus, it is quite likely that pseudogamous and sexual females commonly have more or less separate niches.

From the result of the two extreme cases we have analyzed (identical niches versus non-overlapping niches), it seems reasonable to conclude that the likelihood of stable coexistence decreases as niche overlap decreases. If this is true, our model provides a possible explanation for why pseudogamy is apparently rare in dioecious organisms despite evidence demonstrating that it can evolve in a wide variety of plants and animals. The same result also supports the argument that natural selection should favor rapid loss in pseudogamous lineages of dependence upon sperm.

We have chosen to analyze cases with rather special assumptions on the forms of the basic model-quantities (i.e., $\alpha, \beta_{i}$, $\mu_{i}, p$ and $q$ ). This we have done in order to obtain some improved insight into the general problem of pseudogamy. However, given empirical data on the specific forms of functions like $\alpha=\alpha(z, x, y, x / y)$, $\beta_{i}=\beta_{i}(z, x, y, z / y)$, etc., particular cases of pseudogamy might be analyzed with respect to stability by studying the general model we have suggested. In this way theory and data could be brought together in our efforts to understand the establishment and maintenance of pseudogamy.

The model we have presented and analyzed ought to be further developed and refined in several directions:

1. The model should be refined so as to treat habitat-heterogeneity specifically. Thus, we would study the effects of variable patch sizes as well as the effect of different degrees of hostility of the transition habitat (i.e., the area between breeding places, especially as it is related to migration between patches). By so doing, we might be able to specify under which habitatconfigurations pseudogamy could establish itself and be maintained.

2. The model should be refined so as to incorporate genetic aspects more explicitly. Specifically, the model should be redefined by using a quantitative genetics approach (see e.g., Roughgarden, 1979). We might then be able to specify some of the genetic constraints involved in the evolution of pseudogamy.

3. Using a model as described under item 
2 , we could ask questions about evolutionary consequences of mixed sexual and pseudogamous populations as compared with purely sexual populations. For example, it would be worthwhile-and indeed importantto compare how a mixed population of pseudogamous and sexual females on the one hand, and a purely sexual population on the other hand, respond evolutionarily to changes in the abiotic and/or the biotic component of the environment.

4. The model should be extended to incorporate pseudogamy in hermaphroditic lineages which produce some sperm/pollen. (This problem is not addressed specifically by Kiester et al. [1981], although they do discuss pseudogamy in hermaphroditic organisms.) While this is an unusual situation in animals (but see Christensen, 1980), it seems to be the general case in pseudogamous plants (e.g., Gustafsson 1946-1947 p. 80-84). Competition with related sexual taxa for sperm/pollen may still often occur, however; in plants, this will usually take the form of competition for pollinators, competition which should exist even when pseudogamous lineages do produce fertile pollen.

5. Our model should-along with the relevant empirical information-be analyzed with respect to which pseudogamous strategy (i.e., which combinations of $\beta_{2}$ and $\mu_{2}$ ) is an ESS (as defined by Maynard Smith [1982]). Such an analysis should be done by applying the particular ESS-method developed by Lawlor and Maynard Smith (1976) as extended by Reed and Stenseth (1984).

6. Finally, it is difficult to ignore the accumulating evidence that the pseudogamous portion of a given mixed population is often composed of several clones with differing ecological adaptations (Vrijenhoek, 1978, 1979; Bulger and Schultz, 1979; Mitter et al., 1979; Moore and Eisenbrey, 1979; Christensen, 1980; Schneider, 1980;
Booij, 1981). Such genetic variability may be an important factor in the persistence of pseudogamy, and the model discussed in this paper should be extended to cover such cases.

However, the highest priority should be obtaining more empirical information regarding the demography and behavior of pseudogamous populations. The present model, even though it is a very simplified one, specifies some of the questions needing an answer before further progress-theoretical as well as empirical-is possible.

\section{ACKNOWLEDGMENTS}

W. D. Hamilton helped significantly in early discussions of the model. We thank B. Antonsen for valuable help with the analysis. The manuscript was greatly improved by the comments of J. Felsenstein, W. S. Moore, and an anonymous referee. Financial support for this study was obtained from NATO, the Norwegian and Swedish National Sciences Foundations (NAVF and NFR), the Norwegian Agricultural Science Foundation (NLVF), and the Department of Biology, Division of Zoology of the University of Oslo.

\section{Literature Cited}

ARNold, V. I. 1973. Ordinary Differential Equations. MIT Press, Cambridge.

BAKKE, A. 1968. Field and laboratory studies on sex ratio in Ips acuminatus (Coleoptera: Scolytidae) in Norway. Can. Entomol. 100:640-648.

Bell, G. 1982. The Masterpiece of Nature. Croom Helm, London.

Birky, C. W., AND J. J. Gilbert. 1971. Parthenogenesis in rotifers: the control of sexual and asexual reproduction. Amer. Zool. 11:245-266.

Bool, C. J. H. 1981. Biosystematics of the Muellerianella complex (Homoptera, Delphacidae), taxonomy, morphology and distribution. Neth. J. Zool. 31:572-595.

Boois, C. J. H., AND J. A. GuLdemond. 1984. Distributional and ecological differentiation between asexual gynogenetic planthoppers and related sexual species of the genus Muellerianella (Homoptera, Delphacidae). Evolution 38:163175.

Bulger, A. J., ANd R. J. Schultz. 1979. Heterosis and interclonal variation in the thermal tolerance in unisexual fishes. Evolution 33:848859. 
Christensen, B. 1980 . Constant differential distribution of genetic variants in polyploid parthenogenetic forms of Lumbricillus lineatus (Enchytraeidae, Oligochaeta). Hereditas 92:193198.

Clanton, W. 1934. An unusual situation in the salamander Ambystoma jeffersonianum. Occas. Pap. Mus. Zool. Univ. Mich. 290:1-14.

Drosopoulos, S. 1977. Biosystematic studies on the Muellerianella complex (Delphacidae, Homoptera, Aunchenorrhyncha). Meded. Landbouwhogeschool Wageningen 77:1-133.

- 1978. Laboratory synthesis of a pseudogamous triploid "species" of the genus Muellerianella (Homoptera, Delphacidae). Evolution 32: 916-920.

FOCKE, W. O. 1881. Die Pflanzenmischlinge. Borntraeger, Berlin.

Gustafsson, $\AA$. 1946-1947. Apomixis in higher plants. Lunds Universitets Arsskrift N.F. 4243:1-370.

HaLE, W. G. 1964. Experimental studies on the taxonomic status of some members of the $\mathrm{On}$ ychiurus armatus species groups. Rev. Ecol. Biol. 1:501-510.

Kiester, A. R., T. Nagylaki, and B. Schaffer. 1981. Population dynamics of species with gynogenetic sibling species. Theoret. Pop. Biol. 19:358-369.

Kostitzin, V. A. 1937. Biologie Mathématique. A. Colin, Paris.

LANier, G. N., AND J. H. Oliver. 1966. "Sexratio" condition: unusual mechanism in bark beetles. Science 153:208-209.

LAwLOR, L. R., AND J. MAYNARD SMITH. 1976. The coevolution and stability of competing species. Amer. Natur. 110:79-99.

LewIS, W. H., ed. 1980. Polyploidy: Biological Relevance. Plenum, N.Y.

Maynard Smith, J. 1978. The Evolution of Sex. Camb. Univ. Press, Cambridge.

- 1982. Evolution and the Theory of Games. Camb. Univ. Press, Cambridge.

McKay, F. E. 1971. Behavioral aspects of population dynamics in unisexual-bisexual Poeciliopsis (Pisces: Poeciliidae). Ecology 52:778-790.

Mitter, C., AND D. J. FutuYMa, 1977. Parthenogenesis in the fall cankerworm, Alsophila pometaria (Lepidoptera, Geometridae). Entomol. Exp. Appl. 21:192-198.

Mitter, C., D. J. Futuyma, J. C. Schneider, AND J. D. HARE. 1979. Genetic variation and host plant relations in a parthenogenetic moth. Evolution 33:777-790.

MOORE, W. S. 1976. Components of fitness in the unisexual fish Poeciliopsis monacha occidentalis. Evolution 30:564-578.

MoORE, W. S., AND A. B. EISEnBrey. 1979. The population structure of an asexual vertebrate, Poeciliopsis 2 monachalucida. Evolution 33:563578.

MOORE, W. S., AND F. E. MCKay. 1971. Coexistence in unisexual-bisexual species complexes of Poeciliopsis (Pisces: Poeciliidae). Ecology 52: 791-799.

NyGREN, A. 1954. Apomixis in the angiosperms, II. Bot. Rev. 20:577-649.

OLIVER, J. H. 1971. Introduction to the symposium of parthenogenesis. Amer. Zool. 11:241243.

Pianka, E. R. 1970. On r- and K-selection. Amer. Natur. 104:592-597.

PimM, S. L. 1983. Food webs, food chains and return times. In D. R. Strong and D. S. Simberloff(eds.), Ecological Communities: Conceptual Issues and the Evidence. Princeton Univ. Press, Princeton.

Reed, J., AND N. C. Stenseth. 1984. On evolutionarily stable strategies (ESS). J. Theor. Biol. 108:491-508.

Roughgarden, J. 1979. Theoretical Population Genetics and Evolutionary Ecology: An Introduction. Macmillan, N.Y.

SCHNEIDER, J. C. 1980 . The role of parthenogenesis and female aptery in microgeographic, ecological adaptation in the fall cankerworm, $\mathrm{Al}$ sophila pometaria Harris (Lepidoptera: Geometridae). Ecology 61:1082-1090.

Schultz, R. J. 1969. Hybridization, unisexuality and polyploidy in the teleost Poeciliopsis (Poeciliidae) and other vertebrates. Amer. Natur. 103: 605-619.

-1973. Unisexual fish: laboratory synthesis of a "species." Science 179:180-181.

. 1977. Evolution and ecology of unisexual fishes. Evol. Biol. 10:277-331.

TANNER, J. T. 1966. Effects of population density on growth rates of animal populations. Ecology 47:734-740

Uzzell, T. M., JR., AND S. M. GoldblatT. 1967. Serum proteins of salamanders of the Ambystoma jeffersonianum complex, and the origin of the triploid species of this group. Evolution 21: 345-354.

VRIJENHOEK, R. C. 1978. Coexistence of clones in a heterogeneous environment. Science 199: 549-552.

—_. 1979. Factors affecting clonal diversity and coexistence. Amer. Zool. 19:787-797.

WhITE, M. J. D. 1978. Modes of Speciation. Freeman, San Francisco.

WILBUR, H. M. 1971. The ecological relationship of the salamander Ambystoma laterale to its allfemale, gynogenetic associate. Evolution 25:168179.

Williams, G. C. 1975. Sex and Evolution. Princeton Univ. Press, Princeton.

Corresponding Editor: J. Felsenstein

\section{ApPendix A}

The Jacobian Matrix for (2) evaluated at Equilibrium $\left(x^{*}, y^{*}\right)$.

The Jacobian matrix (see Arnold, 1973) for (2) 
evaluated at the equilibrium $\left(x^{*}, y^{*}\right)$, where $z=$ $2 \cdot x+y$, is defined by

$$
\mathbf{A}=\left(\begin{array}{ll}
a_{11} & a_{12} \\
a_{21} & a_{22}
\end{array}\right)
$$

where

$$
\begin{aligned}
& a_{11}=\left[\frac{d \theta_{1}}{d z} \cdot r+.5 \cdot \theta_{1} \cdot M-2 \cdot \frac{d \mu_{1}}{d z}\right] \cdot x^{*} \\
& a_{12}=\left[.5 \cdot \frac{d \theta_{1}}{d z} \cdot r+.5 \cdot \theta_{1} \cdot N-\frac{d \mu_{1}}{d z}\right] \cdot x^{*} \\
& a_{21}=\left[2 \cdot \frac{d \theta_{2}}{d z} \cdot r \cdot \frac{q}{p}+\theta_{2} \cdot Q-2 \cdot \frac{d \mu_{2}}{d z}\right] \cdot y^{*}
\end{aligned}
$$

and

$$
a_{22}=\left[\frac{d \theta_{2}}{d z} \cdot r \cdot \frac{q}{p}+\theta_{2} \cdot S-\frac{d \mu_{2}}{d z}\right] \cdot y^{*}
$$

where further

$$
\begin{aligned}
M= & \left(2 \cdot \frac{d p}{d z} \cdot y^{*} \cdot x^{*}+p \cdot q \cdot y^{*}\right) \\
& \left(p \cdot x^{*}+q \cdot y^{*}\right)^{2} \\
N= & \left(\frac{d p}{d z} \cdot y^{*} \cdot x^{*}-p \cdot q \cdot x^{*}\right) \\
& \left(p \cdot x^{*}+q \cdot y^{*}\right)^{2} \\
S= & -\left(\frac{d p}{d z} \cdot\left(x^{*}\right)^{2}+q^{2} \cdot x^{*}\right) \\
& \left(p \cdot x^{*}+q \cdot y^{*}\right)^{2}
\end{aligned}
$$

and

$$
\begin{aligned}
Q= & \left(-2 \cdot \frac{d p}{d z} \cdot\left(x^{*}\right)^{2}+q^{2} \cdot y^{*}\right) \\
& \left(p \cdot x^{*}+q \cdot y^{*}\right)^{2}
\end{aligned}
$$

and where finally

$$
r=p \cdot x^{*} /\left(p \cdot x^{*}+q \cdot y^{*}\right) .
$$

\section{Appendix B}

The Routh-Hurowitz Criterion for (2).

A necessary and sufficient condition for local stability - the so-called Routh-Hurowitz criterion (e.g., Arnold, 1973)-is given by

$$
\lambda_{1}+\lambda_{2}=a_{11}+a_{22}<0
$$

and

$$
\lambda_{1} \cdot \lambda_{2}=a_{11} \cdot a_{22}-a_{12} \cdot a_{21},
$$

where $a_{i j}$, for all $i, j$, is defined in Appendix $\mathrm{A}$, and where $\lambda_{1}$ and $\lambda_{2}$ are the two eigenvalues for the system defined by (2) in the main text. Using the expressions for $a_{i j}$ defined in Appendix A, we may write

$$
\begin{aligned}
\lambda_{1}+\lambda_{2}= & {\left[\frac{d \theta_{1}}{d z} \cdot r+.5 \cdot \theta_{1} \cdot M-2 \cdot \frac{d \mu_{1}}{d z}\right] \cdot x^{*} } \\
& +\left[\frac{d \theta_{2}}{d z} \cdot r \cdot \frac{q}{p}+\theta_{2} \cdot S-\frac{d \mu_{2}}{d z}\right] \cdot y^{*}
\end{aligned}
$$

and, after some algebraic manipulations,

$$
\begin{aligned}
\lambda_{1} \cdot \lambda_{2}= & \gamma \cdot\left(2 \cdot \theta_{2} \cdot q \cdot \frac{d \mu_{1}}{d z}-\theta_{1} \cdot p \cdot \frac{d \mu_{2}}{d z}\right) \\
& +r \cdot q \cdot \gamma \cdot\left(\frac{d \theta_{2}}{d z} \cdot \theta_{1}-\frac{d \theta_{1}}{d z} \cdot \theta_{2}\right) \\
& -\frac{d p}{d z} \cdot \frac{\theta_{1} \cdot \theta_{2}}{2} \cdot q \cdot x^{*} \\
& \cdot\left[2 \cdot y^{*} \cdot x^{*}+p \cdot y^{*} \cdot x^{*}\right. \\
& \left.+q \cdot y^{*}+2 \cdot p \cdot\left(x^{*}\right)^{2}\right]
\end{aligned}
$$

where

$$
\gamma=q \cdot\left(\frac{y^{*}}{2}+x^{*}\right) /\left(p \cdot x^{*}+q \cdot y^{*}\right)^{2} .
$$

